Dicle University Journal of Engineering (DUJE)

web: http://dergipark.gov.tr/dumf

Araștırma Makalesi / Research Article

\title{
Konik dişlilerin dayanım analizinde tredgold yaklaşımının ANSYS ile araştırılması
}

\section{Investigation of tredgold approach in strength analysis of bevel gears with ANSYS}

\author{
Gürkan İrsel* \\ ${ }^{1}$ Trakya Üniversitesi Makine Mühendisliği Bölümü, Edirne, \\ gurkanirsel@trakya.edu.tr ORCID: 0000-0003-0828-6560, Tel: (284) 2261217 (2118)
}

\begin{tabular}{|c|c|}
\hline MAKALE BİLGİLERİ & ÖZET \\
\hline Makale geçmişi: & $\begin{array}{l}\text { Konik dişliler, mekanik güç iletimi için endüstriyel makinelerde oldukça yaygın kullanılan makine elemanlarıdır. } \\
\text { Bir cift konik disli genel anlamda, dis dibi eŏilme gerilmesi ve vüzey temas gerilmesi olarak iki tür zorlanmava }\end{array}$ \\
\hline Geliş: 3 Ocak 2020 & maruz kalmaktadır. Bu zorlanmanın hesaplanması optimum tasarımın gerçekleştirilmesi için oldukça önemlidir. \\
\hline Düzeltme: 8 Eylül 2020 & Bu çalışmada, bir konik dişli çiftinin diş dibi eğilme ve temas gerilmesi hesabı sonlu elemanlar yöntemi ile çözüm \\
\hline Kabul: 10 Eylül 2020 & yapan ANSYS 2019R3 ile ve analitik olarak gerçekleştirilmiştir. Analitik hesapta DIN 3991 yöntemi \\
\hline \multirow{7}{*}{$\begin{array}{l}\text { Anahtar kelimeler: } \\
\text { Tredgold, Konik dişli, ANSYS, } \\
\text { DIN 3991, diş dibi eğilme gerilmesi }\end{array}$} & 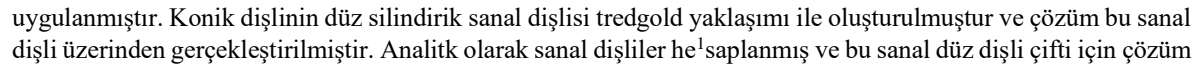 \\
\hline & yapılmıştır. Daha sonra konik dişlilerin katı modelleri CATIA V5 R20 ile oluşturulmuştur. ANSYS mechanical \\
\hline & bölümünde malzeme, temas ve mafsal tanımları yapılmıştır. Dişli çifti için en zor koşul olan tek diş teması için \\
\hline & non-lineer çözüm Newton Rapson metodu ile gerçekleştirilmiştir. Sonuçlar, tredgold yöntemi ile gerçekleştirilen \\
\hline & çözüm ile ANSYS çözümü arasında \% 3.2'lik fark olduğunu göstermiştir. Tredgold yöntemi ile gerçekleştirilen \\
\hline & çözümün standart dişliler için oldukça pratik olduğu, modifiye ve özel dişlilerde ise sonlu elemanlar yönteminin \\
\hline & 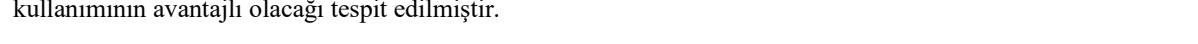 \\
\hline
\end{tabular}

Doi: 10.24012/dumf.669831

\begin{tabular}{ll}
\hline ARTICLE INFO & $\begin{array}{l}\text { ABSTRACT } \\
\text { Article history: }\end{array}$ \\
$\begin{array}{l}\text { Bevel gears are machine elements that are widely used in industrial machines for mechanical power transmission. } \\
\text { A pair of bevel gears is generally subjected to two types of stress: bending stress and surface contact stress. The } \\
\text { calculation of this stress is very important for achieving the optimum design. In this study, the bending and contact } \\
\text { stress calculation of a bevel gear pair was performed analytically with ANSYS 2019R3, which made a solution } \\
\text { wevised: } 8 \text { September } 2020\end{array}$ & $\begin{array}{l}\text { with the finite element method. In the analytical calculation, DIN } 3991 \text { method was applied. The straight } \\
\text { cylindrical virtual gear of the bevel gear was created by the tredgold approach and the solution was realized over } \\
\text { this virtual gear. Virtual gears have been calculated analytically and a solution has been made for this pair of } \\
\text { virtual spur gears Then solid models of bevel gears were created with CATIA V5 R20. Material, contact and joint } \\
\text { definitions were made in ANSYS mechanical section. The non-linear solution for single tooth contact, which is } \\
\text { the most difficult condition for the gear pair, was realized by the Newton Rapson method. The results showed that } \\
\text { there is a 3.2\% difference between the solution realized with the tredgold method and the ANSYS solution. It has } \\
\text { been determined that the solution realized with the Tredgold method is very practical for standard gears, and the } \\
\text { use of the finite element method in modified and special gears will be advantageous. }\end{array}$
\end{tabular}

* Sorumlu yazar / Correspondence

Gürkan IRSEL

$\square$ gurkanirsel@trakya.edu.tr 


\section{Giriş}

Konik dişlilerin optimum tasarımının gerçekleştirilmesi diş dibi eğilme gerilmesi ve yüzey temas gerilmesi hesaplarının doğru yapılmasıyla mümkündür [1] [2]. Dişlilerin arızalanması sadece ekipmanın hasar görmesine neden olmakla kalmamakta, aynı zamanda kişisel güvenlik için de potansiyel risk oluşturmaktadır. Yüzey aşınması zaman içerisinde meydana gelirken, diş dibi kırılmaları ile hasar hızlı bir şekilde ve olumsuz mekanik sorunlar ile sonuçlanmaktadır. Diş dibi kırılma hasarları hatalı kullanım ile şok yüklemelerin, hatalı tasarımların veya hatalı 1sıl işlemin bir göstergesidir. Hasara uğramayacak bir hareket iletim sistemi için dişli hesabı önemlidir.

Dişlilerin prototip üretimleri oldukça maliyetli olduğundan dişlilerin hesapları için teorik ve nümerik hesaplar tercih edilmektedir. Analitik hesabın temeli temeli 1892 y1lında Wilfred Lewis'in geliştirdiği matematiksel modele dayanmaktadır. Lewis, bu eşitliği geliştirirken dişi, bir ankastre cisim olarak ele alarak dişin üzerine etkiyen kuvvetin oluşturduğu maksimum gerilme değerini hesaplamıştır. Ancak, diş dibi yarıçapını, ortaya çıkan gerilme konsantrasyonunu, dinamik etkileri (Hotait \& Kahraman, 2013) ve diş yüklemesinin radyal bileşenini hesaba katmamıştır. 1926'da Timoshenko and Baud fotoelastik bir model geliştirmişleridir [3], Dolan ve Broghamer [4], fotoelastik deneylere dayanan bir gerilme faktörü oluşturarak Lewis'in çalışmalarını geliştirmiştir [5]. Analitik yöntemler bugün bu temel ile tecrübi değerlerin parametreler ile etkitilmesiyle kullanılmaktadır.

Günümüzde dişli hesabı yaygın olarak AGMA 2003 B97, ISO 10300:2001 Method B ve DIN 3991:1988 yöntemleri ile gerçekleştirilmektedir. $\mathrm{Bu}$ yöntemler bu çalışmada sırasıyla kısaca AGMA, ISO, DIN olarak anılacaktır. AGMA, DIN ve ISO standartları ankastre kiriş eğilme gerilmesini temel alan analitik metotlardır.
Analitik yöntemler ile birlikte birlikte 1970'lerden bu yana sonlu elemanlar yöntemiyle de dişli dayanım hesapları gerçekleştirilmektedir. ANSYS, bu sonlu elemanlar yazılımlarından biri ve en yaygın kullanılanıdır [6] [7] [8]

ANSYS ile 3D, 2D tek boyutlu analizler yapılabilmektedir. Üretim için çizilen 3D modellerin ANSYS ile analiz için doğrudan kullanılabilir olması 3D analizlerin kullanımını yaygınlaştırmıştır. $\quad 3 \mathrm{D} \quad$ modelleme modifikasyonların dahil edilmesi ve karmaşık geometrilerin incelenmesine imkan vermesi gibi avantajları olmasına karşın tecrübe gerektiren, karmaşık ve zaman alıcı bir yöntemdir [3]. Analizlerde model, mesh metodu, temas tanımlamaları ve çözdürme yöntemi büyük öneme sahiptir. Düzensiz şekilli ve farklı materyallerden oluşan yapıları modellemek zordur, özellikle de yapı belirli ara yüzler ve ince tabakalar içerdiği takdirde bu durum daha da zorlaşmaktadır. Karmaşık yapıların isabetli FE modellerini geliştirmek zorlayıcı olabilmekte ve karmaşık modellerde kaliteli mesh yapısı üretmek mümkün olmayabilmektedir [9]. Böyle durumlarda mevcut geometrinin uygun analiz işlemleri için düzenlenme çalışmaları yapılması gerekmektedir. Bazı kabuller ile analize uygun modeller oluşturmak bu bağlamda önem kazanmaktadır [10].

Konik dişliler dıştan içe doğru küçülen modül ve değişen diş kalınlığına sahiptirler. Net biçimde koordinatları belirlemek de zahmetli bir işlemdir. $\mathrm{Bu}$ nedenle problemi kolaylaştırmak için konik dişlilere eşdeğer dişli oluşturulmaktadır. Konik dişlilerin analitik hesabında eşdeğer düz dişli Tredgold yaklaşımı ile oluşturulmaktadır [11],[12]. Böylece düz dişlinin hareketi kolaylıkla tanımlanabilmekte ve anlaşılabilmektedir.

\section{Materyal ve Yöntem}

Yapılan bu çalışmada, 21 ve 25 diş sayılarına sahip bir sistemde tredgold yaklaşımı ile eş değer 
düz dişli hesabı ve tasarımı yapılmıştır. Konik dişli çiftinin FEM hesabı gerçekleştirilmiştir. Fem hesabı detaylarına değinilmiş ve doğru sonuç için mesh çalışması açıklanmıştır. Nonlineer sonlu elemanlar çözümü ile tredgold çözüm sonucu karşılaştırılmıştır. Böylece yöntemlerin uygulamalarını ve avantalrını sunan bir çalışma gerçekleştirilmiştir.

\section{Konik Dişlilerin Modellenmesi}

Konik dişliler standartlara göre modellenirler. Konik dişliler bu çalıșmada DIN normuna göre CATIA ile modellenmiştir. Dişliler $90^{\circ}$ açıyla montaj edilmişlerdir.

Dış modülü 4 olan dişliler konik olmaları nedeni ile değişen modüle sahiptirler ve ortalama modül değerleri 3,332' tür. Basınç temas açıları $20^{\circ}$ dir (Tablo1). Tek kademeli bu konik dişli çifti 300 dev/dak hızla $200 \mathrm{Nm}$ tork iletmektir.
Tablo 1. Dişli sistemi geometrik özellikleri

\begin{tabular}{l|c|c|c} 
Diş geometrisi & & Pinyon & Dişli \\
\hline Şaft Açısı $\left(^{\circ}\right)$ & [Sigma] & \multicolumn{2}{|c}{90} \\
Ortalama Modül & [mmn] & \multicolumn{2}{|c}{3.332} \\
Basınç Açısı ( $\left.{ }^{\circ}\right)$ & [alfn] & \multicolumn{2}{|c}{20} \\
Diş Sayısı & {$[\mathrm{z}]$} & 21 & 25 \\
Dişli Genişliği & {$[\mathrm{b}]$} & 25 & 25
\end{tabular}

Dişliler CATIA V5 R20 ile modellenmi v montaj edilmiştir (Şekil 1). Dişlilerin malzemesi 18CrNiMo7-6' dır. Dişli malzeme özellikleri Tablo 2' de görülmektedir.

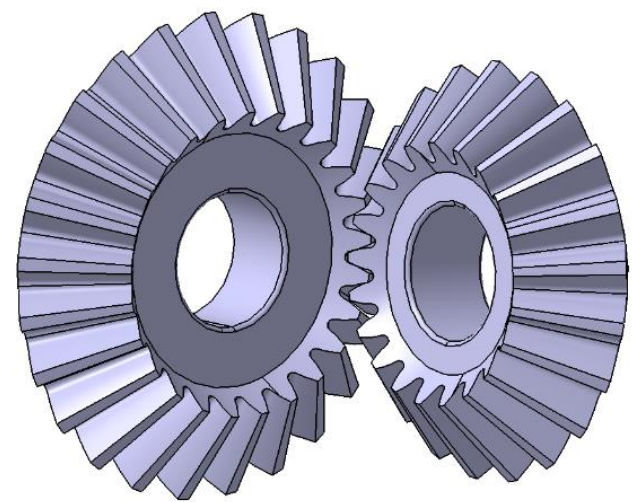

Şekil 1. Konik dişli çifti

Tablo2. Dişli malzeme özellikleri

\begin{tabular}{|c|c|c|c|}
\hline Özellik & & Pinyon & Dişli \\
\hline Dişli malzemesi & & 18CrNiMO7-6 & 18CrNiMO7-6 \\
\hline Yüzey sertliği (HRC) & & 63 & 63 \\
\hline Diş dibi yorulma dayanımı $\left(\mathrm{N} / \mathrm{mm}^{2}\right)$ & {$[\sigma F l i m]$} & 430.00 & 430.00 \\
\hline Yüzey basıncı yorulma dayanımı $\left(\mathrm{N} / \mathrm{mm}^{2}\right)$ & {$[\sigma H l i m]$} & 1500.00 & 1500.00 \\
\hline Çekme gerilmesi $\left(\mathrm{N} / \mathrm{mm}^{2}\right)$ & {$[\sigma B]$} & 1000.00 & 1000.00 \\
\hline Akma gerilmesi $\left(\mathrm{N} / \mathrm{mm}^{2}\right)$ & {$[\sigma S]$} & 695.00 & 695.00 \\
\hline Elastisite modülü $\left(\mathrm{N} / \mathrm{mm}^{2}\right)$ & {$[E]$} & 206000 & 206000 \\
\hline Poisson oranı & {$[v]$} & 0.3 & 0.3 \\
\hline
\end{tabular}

\section{Tredgold Yöntemi}

Tredgold yöntemi; konik dişlinin sanal dişliye indirgenmesi işlemi ve analitik olarak dişlinin daha kolay tanımlanarak çözümlenmesi için gerçekleştirilmektedir [12]. 
Z1 ve Z2 dişlilerinin montajlarının kesitleri montaj açıları ve orta modülden taşınan eksenleri Şekil 2 de görülmektedir. ZV1 ve
ZV2 eşdeğer sanal düz silindirik dişlilerdir. [13].

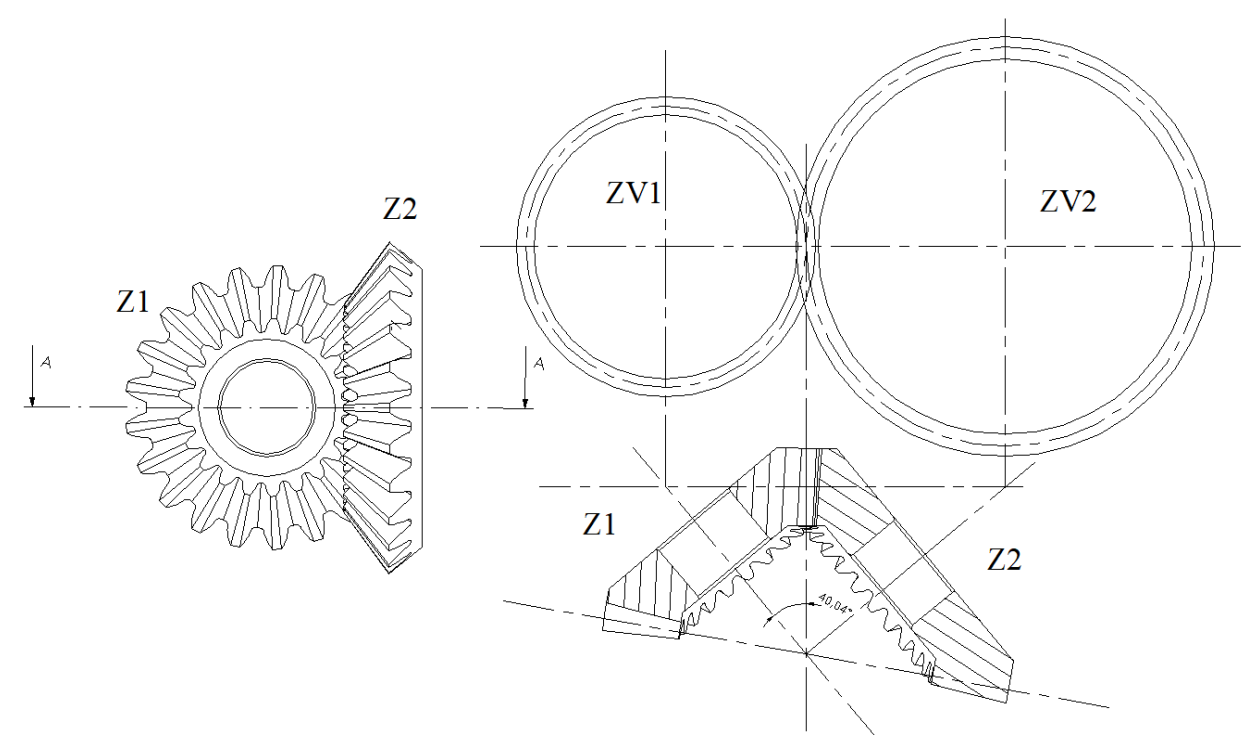

Şekil 2.Tredgold yaklaşımı ile sanal dişlilerin oluşturulması

Tredgold yaklaşımı ile eş değer dişliler (ZV1 ve ZV2) için ortalama modül ve düz dişlilerin boyutları aşağıdaki gibi verilmektedir:

Diş sayıları oranı:

$$
u=\frac{z_{2}}{z_{1}}=\frac{25}{21}=1.19
$$

Çıkış devir sayısı

$$
n_{2}=\frac{n_{1}}{u}=252.10 \mathrm{~d} / \mathrm{d}
$$

Koni tepe Açıları

$$
\begin{aligned}
& \operatorname{tg} \delta_{1}=\frac{1}{u}, \delta_{1}=40,04^{\circ} \\
& \delta_{2}=90-40.04=49.94^{\circ} \\
& M_{1}=200 \mathrm{Nm}
\end{aligned}
$$

Taksimat çapı

$$
\begin{aligned}
& d_{e}=z_{1} \cdot m_{e}\left(m_{e}=4\right) \\
& z_{v 2}=\frac{z_{2}}{\cos \delta_{2}}=38,869
\end{aligned}
$$

Ortalama taksimat çap1

$$
d_{m 1}=d_{e}-b \cdot \sin \delta=69,93 \mathrm{~mm}
$$

Ortalama modül;

$$
m_{m}=3,332
$$

Eşdeğer dişliler

$$
\begin{gathered}
d_{v 1}=\frac{d_{m 1}}{\cos \delta_{1}}=91,328 \\
d_{m 2}=z_{2} \cdot m_{m}=83,325 \mathrm{~mm} \\
d_{v 2}=\frac{d_{m 2}}{\cos \delta_{1}}=129,433 \mathrm{~mm}
\end{gathered}
$$

Nominal kuvvet değeri

$$
F_{m t}=\frac{M}{d_{m 1} / 2}=5720 \mathrm{~N}
$$

Sanal diş sayıları

$$
z_{v 1}=\frac{z_{1}}{\cos \delta_{1}}=27,426
$$

Diş form faktörü $Y_{F}=2.62$, Gerilme düzeltme faktörü $Y_{S}=1.65$, Temas oran1 faktörü $Y_{\varepsilon}=0.70$, Helis açı faktörü $Y_{\beta}=$ 1 'dir (ref). $b^{*}$ efektif diş genişliğidir. 


$$
\begin{gathered}
\sigma_{F 0}=\frac{F_{m t}}{b^{*} m_{e}} Y_{F} \cdot Y_{S} \cdot Y_{\varepsilon} \cdot Y_{\beta} \\
\sigma_{F 0}=244,14 M P a
\end{gathered}
$$

nominal gerilme değeri elde edilir.

\section{ANSYS ile Diş Dibi Gerilme Analizi}

Çalışılan modeldeki dişlinin diş diplerinde oluşan gerilme dağılımını bulabilmek için ANSYS static structural modülünde analiz işlemi gerçekleştirildi. Montaj dosyası ticari CAD yazılımından elde edilerek ANSYS programina import edildi. Space Claim modülü ile geometrilerin topolojileri paylaşıld1. Malzeme özellikleri tanımlanarak yazılıma malzeme kütüphanesinden aktarıldı. Dişlilerin dayanım için en zorlu koşul olan tek dişte teması için birer yüzey temas alanı tanımlandı ve gerilme bu alan üzerinden modellendi.

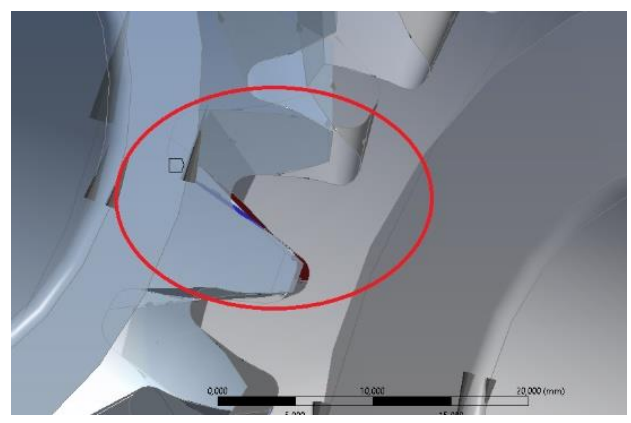

Şekil 3. Diş temas tanımları

Mesh yapılandırma işleminde kullanılacak element modelleme için Hex dominant metod seçildi. Pinyon ve dişli için genel mesh boyutu $1.5 \mathrm{~mm}$ (Şekil 4), yüzeylerde ise mesh boyutu $0,25 \mathrm{~mm}$ olaracak şekilde belirlendi (Şekil 5).

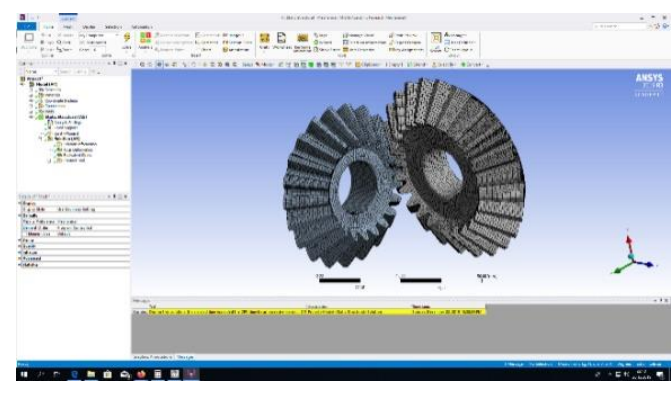

Şekil 4. Mesh ă̆ oluşturulması

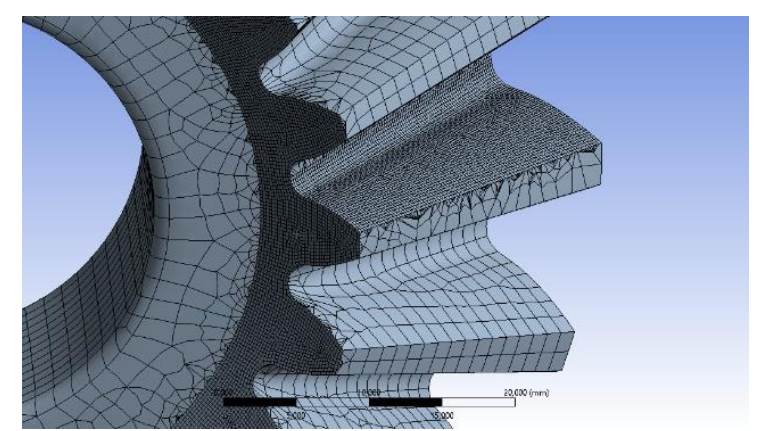

Şekil 5. Temas yüzeyinde mesh yapısı

Yüzeydeki mesh boyutu $0.2 \mathrm{~mm}$, genel çözümde mesh boyutu $1.5 \mathrm{~mm}$ dir. Mesh çarpıklık (skewness) değeri 0.26'dır ve ANSYS mesh değerlendirme kriterine göre 0.25 mükemmel mesh yapısıdır.

Dişli mil deliğinden sabitlenerek pinyon göbeğinde döner mafsal (Revuloint joint) olarak tanımlandı. Bu mafsal üzerinden 200 Nm tork uygulandı (Şekil 6).

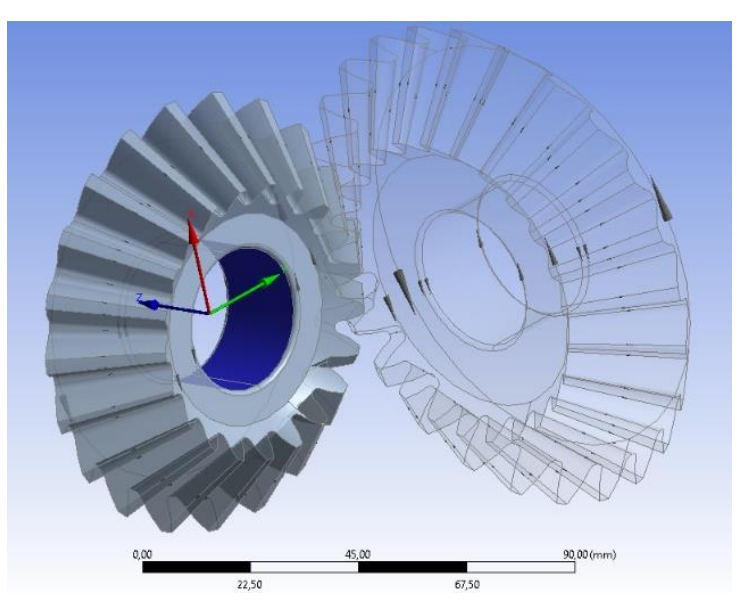

Şekil 6. Sınır şartlarının belirlenmesi

Bir sistemde plastik deformasyona neden olacak gerilme değerlerine ulaşıldığında non-lineer çözüm gerekmektedir. Lineer sonlu elemanlar analizlerinde sisteme bir 
kuvvet uygulandiğında sistemde bir deplasman oluşmaktadır. $\mathrm{Bu}$ deplasman sistemin rijitlik matrisi ile doğru orantılıdır. $\mathrm{Bu} f=k x u$ ile ifade edilir. Ancak nonlineer analizlerde ise sistemin rijitlik matrisi değişkendir. $\mathrm{Bu}$ nedenle çözüm iterasyonlar ile gerçekleştirilir ve bazı kabuller de gerektirir. Şekil değişiminin mukavemete etkisi ve kontaktların değişmesi bu bağlamda önemlidir. $K$ matrisinin deplasman cinsinden yazılması gerekir $\left(\mathrm{K}^{\mathrm{T}}\right.$.)

Non-lineer çözümde yaygın olarak Newton raphson metodu kullanılır. $\mathrm{Bu}$ yöntemde çözüme lineer olarak başlanır. Bir deplasman alanı bulunur. Deplasman vektörü tespit edilip sisteme kuvvet yerine deplasman vektörü uygulanır. Oluşan gerilme sebebiyle iç kuvvetler ile diş kuvvetler birbirine eşit olarak bulunduğunda yakınsama elde edilir. $\mathrm{Bu}$ Convergence (yakınsama) işlemine ait çözüm grafiği Şekil 7' de görülmektedir.

Ansys çözüm işlemi 1 saat 32 dakika sürmüştür. İşlem için 20.7 GB ram kullanılmıştır. 197112 eleman ile çözüm yapılmıştır. $\mathrm{Bu}$ işlemler $4114 \mathrm{CPU} 2.2$ GHZ işlemci ile ANSYS Academic Research Mechanical sürümü ile gerçekleştirilmiştir. Analiz işlemi sonucunda maksimum gerilme değeri diş dibinde $251.96 \mathrm{MPa}$ olarak bulunmuştur (Şekil 8).
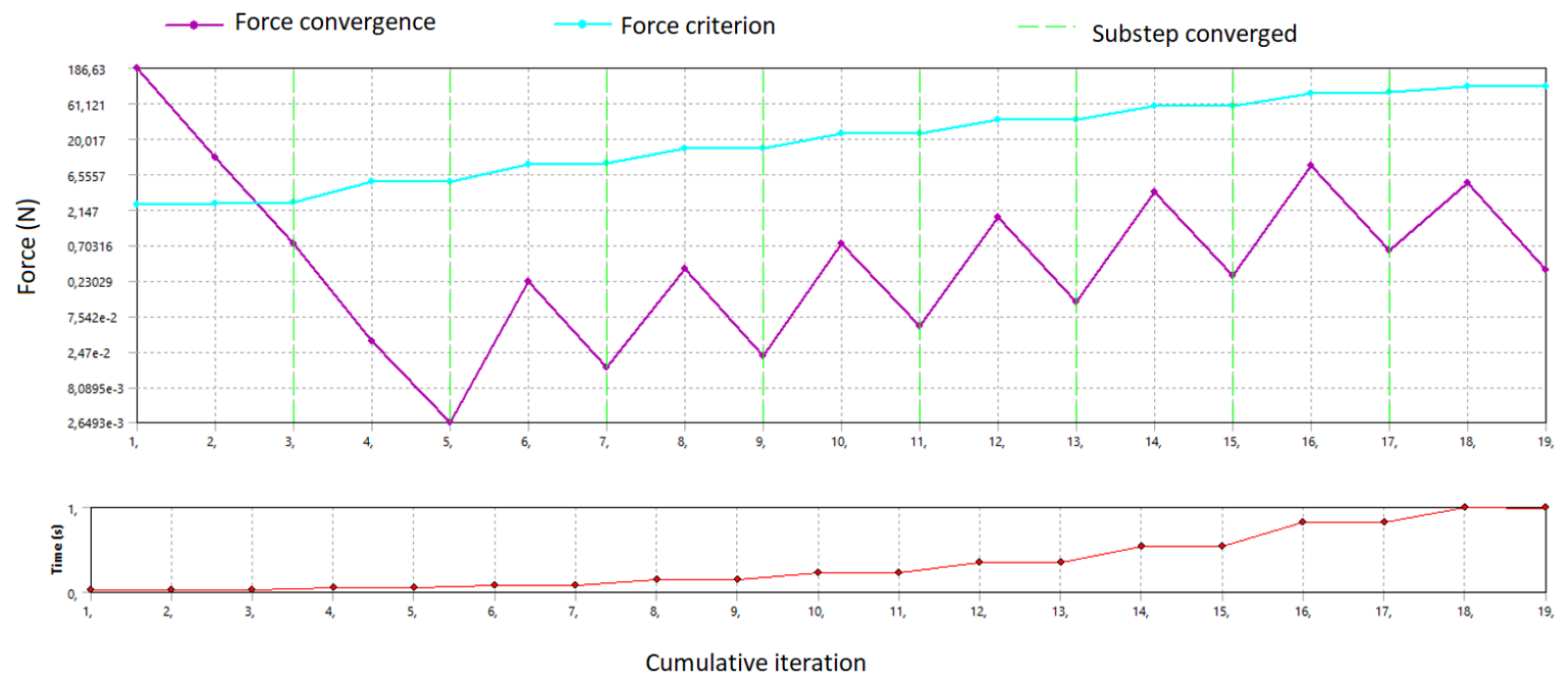

Şekil 7. Birikimli iterasyon işlemi 


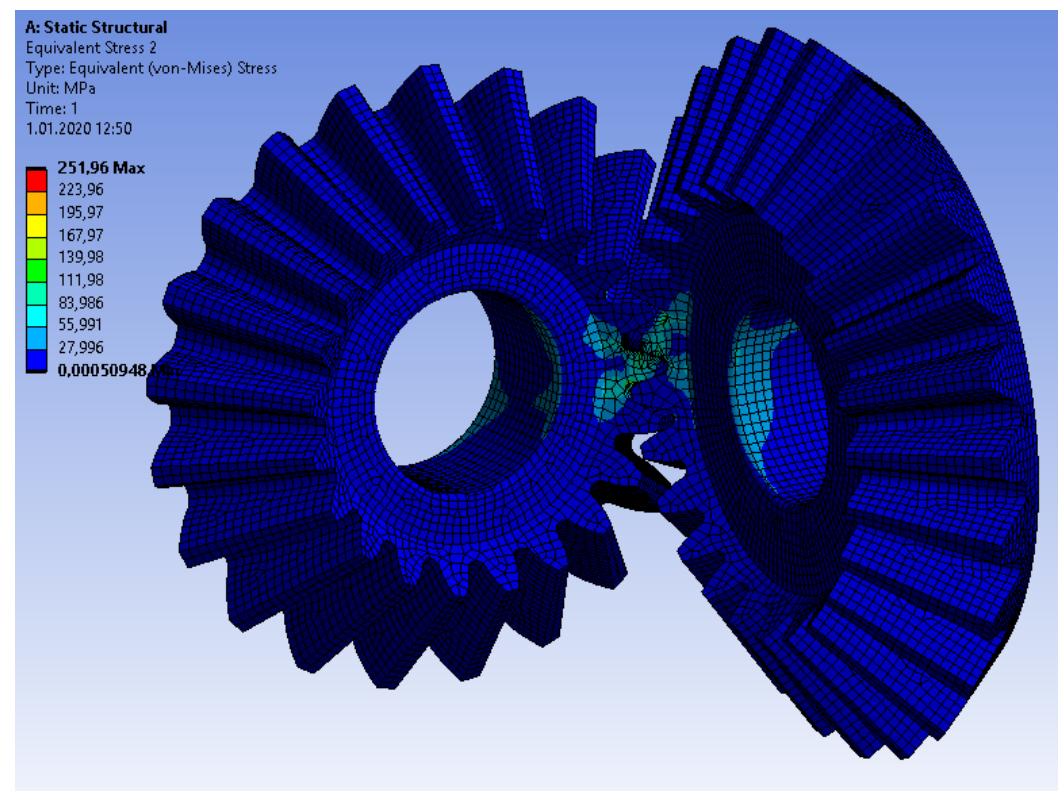

Şekil 8. ANSYS yapısal gerilme analizi

\section{Sonuçlar:}

Yapılan bu çalışmada, tredgold yaklaşımı ile analitik dişli hesabı ile konik dişli çiftinin nümerik hesabının gerçekleştirilmesi, hesaplama işlemlerinin birbirlerine göre avantajları ve sonuçların ilişkisi araştırılmıştır. Aşağıdaki sonuçlar elde edilmiştir.

- Tredgold yaklaşımı ile sanal düz silindirik dişliler kullanılarak gerçekleştirilen analitik hesap ile ANSYS ile gerçekleştirilen nümerik analiz sonuçları oldukça yakındır. Sonuçlar arasında \% 3.2 lik bir fark vardır.

- ANSYS dişli hesabı için özel olarak geliştirilmiş bir yazılım değildir. Maksimum gerilme değerlerinin tespiti yüksek hesaplama kapasiteli bilgisayarlar ve tecrübe gerektirmektedir.

- Analitik hesap elle yaklaşık 15 dakika sürmüştür. Nümerik çözümde katı model oluşturulması, ANSYS tanımlamaları ve tekrarlı çözüm işlemleri ile işlem yaklaşık 8 saat sürmüştür.
- Araştırma sonucunda bilgisayar destekli mühendislik programlarının dişli analizinde oldukça başarılı sonuçlar elde edilebileceğini ortaya koymuştur.

- FEM tabanlı yazılımlar için çözüm prosedürü iyi tanımlandığında otomatikleştirilmiş çözümler gerçekleştirilebilir.

- ANSYS analizinde dinamik faktörler (Kv), Pik yükler (KA) dikkate alınmaz.

- ANSYS maksimum gerilmenin konumunu ve dağılımını sunar ancak analitik hesapta sadece maksimum gerilme değeri tespit edilmektedir.

- Tredgold yöntemi analitik olarak konik dişli sisteminin basit çözümüne imkan sağlamıştır. 


\section{Kaynaklar}

[1] J. Zhan and M. Fard, "Effects of helix angle, mechanical errors, and coefficient of friction on the time-varying tooth-root stress of helical gears," Meas. J. Int. Meas. Confed., vol. 118, no. January, pp. 135-146, 2018.

[2] O. Doğan and F. Karpat, "Crack detection for spur gears with asymmetric teeth based on the dynamic transmission error," Mech. Mach. Theory, vol. 133, pp. 417-431, 2019.

[3] T. J. Lisle, B. A. Shaw, and R. C. Frazer, "External spur gear root bending stress: A comparison of ISO 6336:2006, AGMA 2101D04, ANSYS finite element analysis and strain gauge techniques," Mech. Mach. Theory, vol. 111, pp. 1-9, 2017.

[4] T. J. Dolan and E. L. Broghamer, "A photoelastic study of stresses in gear tooth profiles," Bulletin, no. $335,1942$.

[5] P. R. N. Childs, Spur and helical gear stressing. 2018.

[6] K. N. Naik and D. Dolas, "Static Analysis Bending Stress on Gear Tooth Profile By Variation of Gear Parameters With The Help of FEA," vol. 3, no. 6, pp. 132-137, 2014.

[7] S. Jyothirmai, R. Ramesh, T. Swarnalatha, and D. Renuka, "A Finite Element Approach to Bending, Contact and Fatigue Stress Distribution in Helical Gear Systems," Procedia Mater. Sci., vol. 6, no. Icmpc, pp. 907-918, 2014.
[8] G. Mehta, M. Somani, T. Narendiranath Babu, and T. Watts, "Contact Stress Analysis on Composite Spur Gear using Finite Element Method," Mater. Today Proc., vol. 5, no. 5, pp. 13585-13592, 2018.

[9] X. Liu, Y. Yang, and J. Zhang, "Investigation on coupling effects between surface wear and dynamics in a spur gear system," Tribol. Int., vol. 101, pp. 383-394, 2016.

[10] F. Zheng, M. Zhang, W. Zhang, R. Tan, and X. Guo, "On the deformed tooth contact analysis for forged bevel gear modification," Mech. Mach. Theory, vol. 135, pp. 192-207, 2019.

[11] A. Bahrami Ghahnavieh, S. Akbarzadeh, and P. Mosaddegh, "A numerical study on the performance of straight bevel gears operating under mixed lubrication regime," Mech. Mach. Theory, vol. 75, pp. 27-40, 2014.

[12] D. B. Dooner, M. Vivet, and D. Mundo, "Deproximating Tredgold's Approximation," Mech. Mach. Theory, vol. 102, pp. 36-54, 2016.

[13] M. Vivet, D. Mundo, T. Tamarozzi, and W. Desmet, "An analytical model for accurate and numerically efficient tooth contact analysis under load, applied to face-milled spiral bevel gears," Mech. Mach. Theory, vol. 130, pp. 137-156, 2018. 